\title{
Two muscular variations in the elbow associated with the anterior interosseous nerve
}

\author{
Łukasz Olewnik $^{1}$ (1) $\cdot$ Bartłomiej Szewczyk ${ }^{1} \cdot$ Nicol Zielinska $^{1} \cdot$ Dariusz Grzelecki $^{3} \cdot$ Michał Polguj $^{2}$
}

Received: 4 November 2020 / Accepted: 2 February 2021 / Published online: 15 February 2021

(c) The Author(s) 2021

\begin{abstract}
The coexistence of different muscular-neurovascular variations is of significant clinical importance. A male cadaver, 76 years old at death, was subjected to routine anatomical dissection; the procedure was performed for research and teaching purposes at the Department of Anatomical Dissection and Donation, Medical University of Lodz. The right forearm and hand were dissected using standard techniques according to a strictly specified protocol. The presence accessory head of the flexor pollicis longus may potentially compress the anterior interosseous nerve. The present case report describes a rare variant of the ulnar head of the pronator teres, characterized by two independent bands (i.e., two proximal attachments). The main band originates from the coronoid process and the second originates from the tendon of the biceps brachii. This type of attachment could potentially affect the compression of the ulnar artery running between the two bands. Additionally, the accessory head of the flexor pollicis longus was observed, which started on the medial epicondyle; its coexistence with a high division median nerve creates a potential pressure site on the anterior interesosseous nerve.
\end{abstract}

Keywords Flexor pollicis longius $\cdot$ Pronator teres $\cdot$ Anterior interosseous nerve $\cdot$ Median nerve $\cdot$ Case report

\section{Introduction}

Morphological variations can affect routine clinical procedures or important and complex surgeries. For example, the occurrence of a coracobrachialis longus muscle may influence potential compression on the median nerve, ulnar nerve or musculocutaneous nerve [22, 23]. A similar situation can

Łukasz Olewnik

lukasz.olewnik@umed.lodz.pl

Bartłomiej Szewczyk

bartlomiej.szewczyk@umed.lodz.pl

Nicol Zielinska

nicol.zielinska@stud.umed.lodz.pl

Dariusz Grzelecki

dariusz.grzelecki@gmail.com

Michał Polguj

michal.polguj@umed.lodz.pl

1 Department of Anatomical Dissection and Donation, Medical University of Lodz, Łódź, Poland

2 Department of Normal and Clinical Anatomy, Medical University of Lodz, Łódź, Poland

3 Department of Orthopedics and Rheumooorthopedics, Centre of Postgraduare Medical Education, Otwock, Poland occur at the site of the median nerve ran between the two pronator teres heads, as well as the site where the median nerve crosses the palmaris longus tendon [20,21]. The accessory head of the flexor pollicis longus (ahFPL) may lead to entrapment neuropathy of the anterior interosseous nerve (AIN) [1, 2, 6, 9, 13, 15, 19, 25].

Earlier studies on the morphological variability of the pronator teres (PT) focused mainly on the incidence of the ulnar head $[7,11,14,18,21,28]$ or its relationship to the median nerve [ $7,11,14,18,21,28]$. In contrast, variations in the flexor pollicis longus mainly concerned its morphological variability, and studies focused on the incidence of composite accessory heads and place of origin $[1,2,6,9$, $13,15,19,25]$.

This paper describes an extremely rare co-occurrence of a rare variation of the PT ulnar head (uhPT) with one of the accessory head of the flexor pollicis longus (ahFPL). The dual proximal attachment of the uhPT creates a potential pressure point on the ulnar artery. It should be noted that the prevalence of vascular compression in the elbow area is extremely rare: only $1 \%$. Our findings highlight the importance of muscle variants in the forearm region, and are of interest to radiologists, anatomists, physiotherapists and surgeons specializing in the forearm and hand region. 


\section{Case report}

A male cadaver, 76 years old at death, was subjected to routine anatomical dissection for research and teaching purposes at the Department of Anatomical Dissection and Donation, Medical University of Lodz. The right forearm and hand were dissected using standard techniques according to a strictly specified protocol [20, 21, 30, 31]. During the dissection of the left forearm, two morphological variations were found.

1. Accessory head of the flexor pollicis longus muscle (ahFPL):

The ahFPL originated from the medial epicondyle of the humerus, under the flexor digitorum superficialis. The origin began with a thin, 78.04 mm-long tendon. The origin of the accessory head was fused with most of the brachialis muscle, as well as the ulnar head of the pronator teres muscle. The proximal tendon passed into a $54.80 \mathrm{~mm}$-long muscle belly; the belly then passed into a $54.14 \mathrm{~mm}$-long distal tendon and inserted on the radial border of the FPL. It was fusiform-shaped-Fig. 1.

2. A rare case of the ulnar head of the pronator teres (uhPT) and its relationship to the ulnar artery

The PT consisted of two independent heads (the humerus and ulna) that did not connect to one another in the distal part. The humeral head was cut to highlight the variability regarding the ulnar head of the PT. The ulnar head originates by two independent bands (two proximal attachments). The main band originated from the coronoid process and the accessory bands from the AHFPL, while the second originated from the tendon of the biceps brachii-Fig. 1. Morphometric measurements of the bands are given in Table 1. Following this, both bands joined together and became the muscle belly; the distal tendon then inserted at the middle of the lateral surface of the radius. A "fibrous arch" can be seen at the junction of the two bands, through which the ulnar artery runs. At the transition point, it has a diameter of $5.09 \mathrm{~mm}$-Fig. 1 .

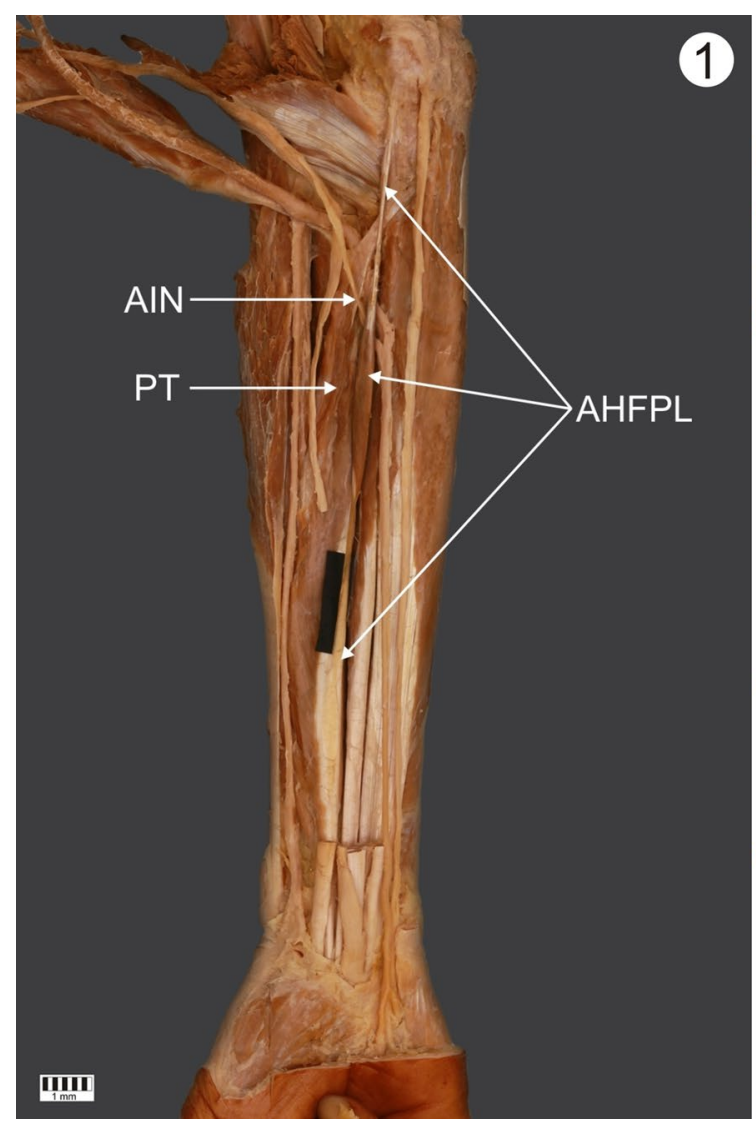

Fig. 1. 1 Complete view of the forearm. Right forearm. Anterior view. Visible accessory head of the flexor pollicis longus. AHFPL accessory head of the flexor pollicis longus. $P T$ pronator teres muscle AIN anterior interosseous nerve. 2 Proximal part of the forearm.

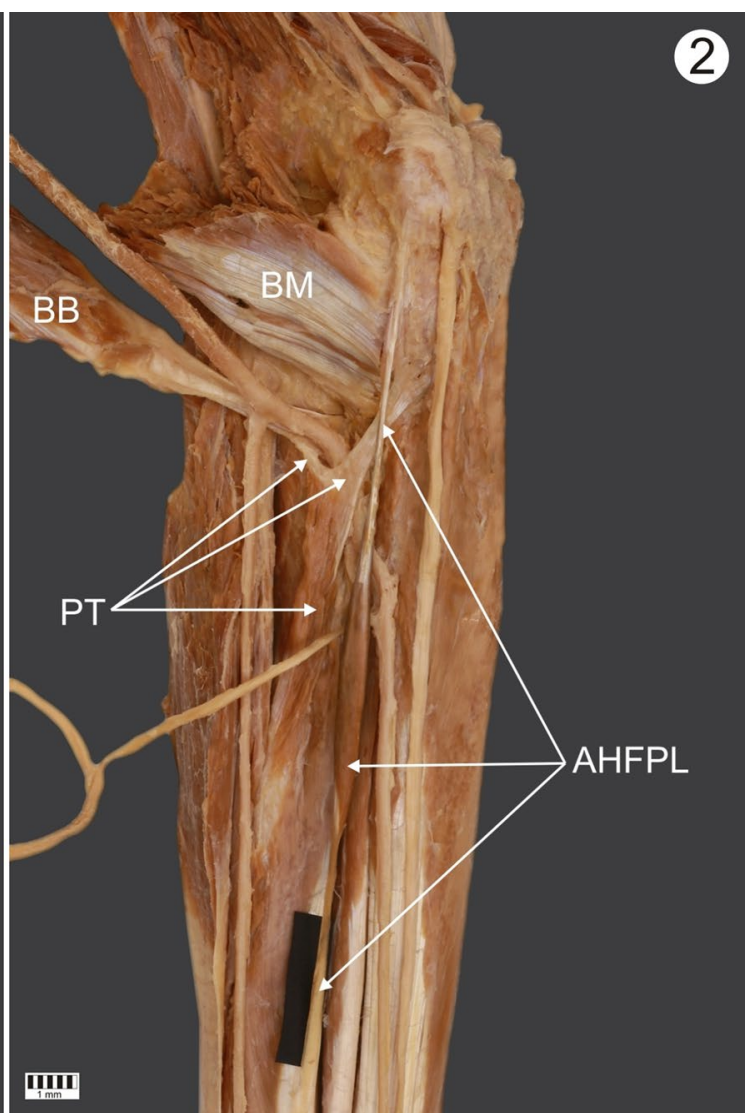

Anterior view of the right forearm. $B M$ brachialis muscle $B B$ biceps brachii $A H F P L$ accessory head of the flexor pollicis longus $P T$ pronator teres muscle 
Table 1 Morphometric measurements of the proximal attachments of the ulnar head of the PT

\begin{tabular}{lll}
\hline & \multicolumn{1}{l}{ Origin } \\
\cline { 2 - 3 } & First band & Second band \\
\hline & Coronoid process & $\begin{array}{c}\text { Tendon of } \\
\text { the biceps } \\
\text { brachii }\end{array}$ \\
Length & $29.13 \mathrm{~mm}$ & $8.42 \mathrm{~mm}$ \\
Tendinous part & & \\
Width & $6.17 \mathrm{~mm}$ & $2.59 \mathrm{~mm}$ \\
Origin & $8.19 \mathrm{~mm}$ & $4.54 \mathrm{~mm}$ \\
Myotendinous junction & & $1.45 \mathrm{~mm}$ \\
Thickness & $3.37 \mathrm{~mm}$ & $1.67 \mathrm{~mm}$ \\
Origin & $4.01 \mathrm{~mm}$ & \\
Myotendinous junction & &
\end{tabular}

The measurements were performed using two methods:

- Direct measurements by electronic caliper (Mitutoyo Corporation, Kawasaki-shi, Kanagawa, Japan). Each measurement was carried out twice with an accuracy of up to $0.1 \mathrm{~mm}$.

- Analysis of digital photographic images processed through MultiScanBase 18.03 (Computer Scanning System II, Warsaw, Poland).

\section{Discussion}

Our case illustrates an extremely rare co-occurrence of a rare variety of PT ulnar head and an accessory head of the flexor pollicis longus. The uhPT originates by two independent bands (two proximal attachments): the main one originates from the coronoid process and the other from the biceps brachii tendon. This arrangement is associated with a potential risk of compression on the ulnar artery. The ahFPL originates from the medial epicondyle of the humerus, under the flexor digitorum superficialis.

Morphological variations in PT occur quite frequently and most often concern the presence or absence of uhPT or the relationship of the whole muscle to the course of the MN $[4,7,11,21,26,28]$. The frequency of occurrence of the uhPT has been found to vary greatly between studies. Its absence has been noted in $10.8 \%$ of cases in one study[11], in $15 \%$ in another [26], as well as in $22 \%$ [18], in 14\% [7] [21], and in one out of 30 cases [4]. Interestingly, none of these studies noticed the lack of a humeral head of the PT $[4,7,11,14,18,21,26,28]$. Testut [28] and LeDouble [14] report that the humeral head of the PT may be completely separate. The PT was found to present typical independent heads in the present case. Cases involving the third head of the PT have also been described in the literature [3]. The course of the MN in relation to PT depends on
Table 2 Comparison of the course of the median nerve in relation to the pronator teres muscle

\begin{tabular}{lllll}
\hline $\begin{array}{l}\text { Course of median } \\
\text { nerve }\end{array}$ & Mori & $\begin{array}{l}\text { Nebot- } \\
\text { Cegarra } \\
\text { et al. }\end{array}$ & $\begin{array}{l}\text { Jamie- } \\
\text { son and } \\
\text { Anson }\end{array}$ & Olewnik et al. \\
\hline Between both heads & $95 \%$ & $75 \%$ & $83.3 \%$ & $74 \%$ \\
Beneath both heads & $0.25 \%$ & - & $6 \%$ & $12 \%$ \\
Beneath HH & - & $21.6 \%$ & $8.7 \%$ & $14 \%$ \\
Through UH & - & $3.4 \%$ & - & - \\
$\begin{array}{l}\text { Duplicate HH, } \\
\text { through HH }\end{array}$ & $0.25 \%$ & $1.7 \%$ & $2 \%$ & - \\
Together with UA & - & - & - & - \\
\hline
\end{tabular}

$H H$ humeral head, $U H$ ulnar head, $U A$ ulnar artery

the morphology of the muscle and its adjacent anatomical structures (Table 2) [11, 17, 18, 21].

The uhPT originates by two independent bands (two proximal attachments). The main band originates from the coronoid process and the second from the biceps brachii tendon; the latter can compress the ulnar artery. Interestingly, entrapment syndrome is very rare in this region. The prevalence of vascular compression in the elbow area is only $1 \%$ $[5,8,27]$. Talha et al. [27] describe a case of brachial artery entrapment caused by compression by the supracondylar process, Blakeborough et al. [5] report ulnar artery entrapment by the ligament of Struthers, while Chemla et al. [8] describe two cases of forearm arterial entrapment syndrome in patients having angioaccesses for hemodialysis: one case was due to a fibrosis arcade arising from the flexor digitorum superficialis muscle and the other was due to pronator teres muscle syndrome. Both entrapment syndromes were revealed by angioaccess iterative thromboses [8]. Talha et al. [27], Blakeborough et al. [5] and Chemla et al. [8] report no neurological complaints during the compression.

In the present case reports, the uhPT was characterized by the presence of two proximal attachments with a "fibrous arch" formed at the junction of the two bands; this fibrous arch provides passage for the ulnar artery. At the transition point, it has a diameter of $5.09 \mathrm{~mm}$. This is a potential site of compression on the ulnar artery. But as vascular compression is largely asymptomatic, it is rarely reported.

Despite playing an important role in the potential compression on nerves, arteries or veins, muscular coexistence is very rarely described in the literature [23, 24]. The present report describes the coexistence of a rare uhPT variety with an accessory head of the flexor pollicis longus. The presence of this uhPT type, i.e., originating with a single band from the biceps brachii, may shift the contraction force from the biceps brachii over the elbow joint, thus helping the biceps brachii flex the elbow. The ahFPL may originate from the medial epicondyle of the humerus, the coronoid process of the ulna or the flexor digitorum superficialis muscle $[2,9$, 
$10,16,29]$. In the present case report, the ahFPL was found to originate from the medial epincondyle, under the flexor digitorum superficialis. The incidence of the ahFPL is quite high and ranges from 25 to $73.6 \%$. [2, 9, 10, 12, 16, 29]. The ahFPL arises during the development of the common flexor mass. This differentiates into superficial and deep layers, the latter differentiating into the FDP, the flexor pollicis longus and the pronator quadratus muscle. Incomplete cleavage of the deep layer results in the formation of the ahFPL. The FPL is primitive or absent in primates, due to it being incorporated into the flexor muscle mass; in such cases the thumb flexor has no functional independence.

Anterior Interosseous nerve (AIN) syndrome is a rare syndrome that comprises less than $1 \%$ of all upper extremity nerve palsies, arising due to compression or inflammation of the AIN of the forearm. The ahFPL may lead to entrapment neuropathy of the AIN. This syndrome is manifested clinically as a weakness in the flexion of the interphalangeal joint of the thumb and the distal interphalangeal joints of the index and middle finger. In the present case report, the AIN ran under the ahFPL in the distal part; this is also a potential site for nerve compression.

\section{Conclusion}

The coexistence of the rare ulnar head of pronator with the accessory head of the flexor pollicis longus are extremely rare. The presence of a double proximal attachment of the pronator teres may additionally put pressure on the ulnar artery.

\footnotetext{
Author contributions Łukasz Olewnik (D.P.T., PhD)—assistant-project development, data collection and management, data analysis and manuscript writing. Bartłomiej Szewczyk - assistant—data analysis, manuscript editing. Nicol Zielinska-assistant—data analysis, manuscript editing. Dariusz Grzelecki (MD, PhD)—assistant—data analysis, manuscript editing. Michał Polguj (MD., PhD)—professor-data analysis, manuscript editing. All authors have read and approved the manuscript.
}

Funding The authors have no financial or personal relationship with any third party whose interests could be positively or negatively influenced by the article's content. This research did not receive any specific grant from funding agencies in the public, commercial, or not-for-profit sectors.

Data availability Please contact authors for data requests (Łukasz Olewnik PhD—email address: lukasz.olewnik@umed.lodz.pl).

\section{Compliance with ethical standards}

Conflict of interest The authors declare that they have no competing interests.
Ethical approval The cadaver belonged to the Department of Anatomical Dissection and Donation, Medical University of Lodz.

Open Access This article is licensed under a Creative Commons Attribution 4.0 International License, which permits use, sharing, adaptation, distribution and reproduction in any medium or format, as long as you give appropriate credit to the original author(s) and the source, provide a link to the Creative Commons licence, and indicate if changes were made. The images or other third party material in this article are included in the article's Creative Commons licence, unless indicated otherwise in a credit line to the material. If material is not included in the article's Creative Commons licence and your intended use is not permitted by statutory regulation or exceeds the permitted use, you will need to obtain permission directly from the copyright holder. To view a copy of this licence, visit http://creativecommons.org/licenses/by/4.0/.

\section{References}

1. Al-Qattan MM (1996) Gantzer's muscle: an anatomical study of the accessory head of the flexor pollicis longus muscle. J. Hand Surg (British Eur) 21(2):269-270. https://doi.org/10.1016/S0266 -7681(96)80114-8

2. Ballesteros DR et al (2019) Accessory head of the flexor pollicis longus muscle: Anatomical study and clinical significance. Folia Morphol 78(2):394-400. https://doi.org/10.5603/FM.a2018.0091

3. Barrett JH (1936) An additional (third and separate) head of the pronator teres muscle. J Anat 70(Pt 4):577-578

4. Bilecenoglu B et al (2005) Possible anatomic structures causing entrapment neuropathies of the median nerve: an anatomic study. Acta Orthop Belg 71:169-176

5. Blakeborough A et al (1994) Case report: Cellist's elbow?-Vascular entrapment in association with the ligament of struthers. Clin Radiol 49(12):902-904. https://doi.org/10.1016/S0009 $-9260(05) 82891-0$

6. Caetano EB et al (2015) Gantzer muscle. An anatomical study. Acta Ortop Bras 23(2):72-75. https://doi.org/10.1590/1413-78522 015230200955

7. Caetano EB et al (2017) Anatomical variations of pronator teres muscle: predispositional role for nerve entrapment. Rev Bras Ortop 52(2):169-175. https://doi.org/10.1016/j.rboe.2017.02.003

8. Chemla ES et al (2001) Forearm arteries entrapment syndrome: a rare cause of recurrent angioaccess thrombosis. J Vasc Surg 34(4):743-747. https://doi.org/10.1067/mva.2001.116973

9. El Domiaty MA et al (2008) The prevalence of accessory heads of the flexor pollicis longus and the flexor digitorum profundus muscles in Egyptians and their relations to the median and anterior interosseous nerves. Folia Morphol (Warsz) 67(1):63-71

10. Hemmady MV et al (1993) Occasional head of flexor pollicis longus muscle: a study of its morphology and clinical significance. $\mathrm{J}$ Postgrad Med 39(1):14-16

11. Jamieson RW, Anson BJ (1952) The relation of the median nerve to the heads of origin of the pronator teres muscle, a study of 300 specimens

12. Jones $M$ et al (1997) Incidence and morphology of accessory heads of flexor pollicis longus and flexor digitorum profundus (Gantzer's muscles). J Anat 191(3):451-455. https://doi.org/10.1 046/j.1469-7580.1997.19130451.x

13. Kara A et al (2012) Accessory head of flexor pollicis longus muscle in fetuses and adult cadavers and its relation to anterior interosseous nerve. Clin Anat 25(5):601-608. https://doi.org/10.1002/ ca. 21296 
14. Le Double A (1897) Traité des variations du système musculaire de l'homme et de leur signification au point de vue de l'anthropologie zoologique. 2: 99-107. Schleicher frères, Paris

15. Mahakkanukrauh $P$ et al (2004) Prevalence of accessory head of flexor pollicis longus muscle and its relation to anterior interosseous nerve in Thai population. Clin Anat 17(8):631-635. https ://doi.org/10.1002/ca.20016

16. Mangini U (1960) Flexor pollicis lungus muscle. Its morphology and clinical significance. J Bone Jt Surg Am 42A:467-470. https ://doi.org/10.2106/00004623-196042030-00010

17. Mori M (1964) Statistics on the musculature of the Japanese. Okajimas Folia Anat Jpn 40:195-300

18. Nebot-Cegarra J et al (1991) Variations of the pronator teres muscle: predispositional role to median nerve entrapment. Arch Anat Histol Embryol 74:35-45

19. Oh CS et al (2000) Anatomical study of the accessory head of the flexor pollicis longus and the anterior interosseous nerve in Asians. Clin Anat 13(6):434-438. https://doi.org/10.1002/10982353(2000)13:6\%3c434::AID-CA7\%3e3.0.CO;2-4

20. Olewnik $€$ et al (2017) Anatomical variations of the palmaris longus muscle including its relation to the median nerve-a proposal for a new classification. BMC Musculoskelet Disord 18(1):1-9. https://doi.org/10.1186/s12891-017-1901-x

21. Olewnik $€$ et al (2018) Anatomical variations of the pronator teres muscle in a Central European population and its clinical significance. Anat Sci Int 93(2):299-306. https://doi.org/10.1007/s1256 5-017-0413-y

22. Olewnik, Ł. et al. (2020a) The co-occurrence of a four headed coracobrachialis muscke, split coracoid process and tunnel for the median and musculocutaneous nerve: the potential clinical relevance of a very rare variation

23. Olewnik $€$ et al (2020b) Potential compression of the musculocutaneous, median and ulnar nerves by a very rare variant of the coracobrachialis longus muscle. Folia Morphol (Warsz). https:// doi.org/10.5603/fm.a2020.0085
24. Olewnik $€$ et al (2020c) The co-occurrence of a four headed coracobrachialis muscke, split coracoid process and tunnel for the median and musculocutaneous nerve: the potential clinical relevance of a very rare variation. Surg Radiol Anat. https://doi. org/10.1007/s00276-020-02580-x

25. Pai MM et al (2008) The accessory heads of flexor pollicis longus and flexor digitorum profundus: Incidence and morphology. Clin Anat 21(3):252-258. https://doi.org/10.1002/ca.20612

26. Stabille $S$ et al (2008) Pronator teres muscle: anatomical variations and predisposition for the compression of the median nerve. Acta Sci Biol Sci 24:631-637

27. Talha $\mathrm{H}$ et al (1987) Brachial artery entrapment: compression by the supracondylar process. Ann Vasc Surg 1(4):479-482. https:// doi.org/10.1016/S0890-5096(06)60736-X

28. Testut L (1884) Les anomalies musculaires chez l'homme: expliquées par l'anatomie comparée leur importance en anthropologie. G. Masson, Paris, pp 454-489

29. Uyaroglu FG et al (2006) Incidence and morphology of the accessory head of the flexor pollicis longus muscle (Gantzer's muscle) in a Turkish population. Neurosciences 11(3):171-174. https:// doi.org/10.1016/j.jasi.2017.08.160

30. Zielinska $\mathrm{N}$ et al (2020a) A very rare case of an accessory subscapularis muscle and its potential clinical significance. Surg Radiol Anat. https://doi.org/10.1007/s00276-020-02531-6

31. Zielinska $\mathrm{N}$ et al (2020b) The subscapularis tendon: a proposed classification system. Ann Anat Anat Anzeiger. https://doi. org/10.1016/j.aanat.2020.151615

Publisher's Note Springer Nature remains neutral with regard to jurisdictional claims in published maps and institutional affiliations. 